EPJ Web of Conferences 60, 18003 (2013)

DOI: $10.1051 /$ epjconf $/ 20136018003$

(C) Owned by the authors, published by EDP Sciences, 2013

\title{
Search for Stop Pair Production at the LHC using the CMS Detector
}

\author{
Verena I. Martinez Outschoorn ${ }^{1, a}$, for the CMS Collaboration \\ ${ }^{1}$ Fermi National Accelerator Laboratory ${ }^{b}$, Batavia, IL, USA
}

\begin{abstract}
A search for pair production of top squark events in proton-proton collisions at a center-of-mass energy of $\sqrt{s}=8 \mathrm{TeV}$ is performed in a sample with a single isolated electron or muon, jets, large missing transverse energy and large transverse mass. The data sample used corresponds to an integrated luminosity of $19.5 \mathrm{fb}^{-1}$ collected by the CMS experiment. No significant excess in data is observed above the expectation from standard model processes. In the context of supersymmetric models with pair production of top squarks decaying to either a top quark and the lightest supersymmetric particle (LSP) or a bottom quark and a chargino that subsequently decays to a $\mathrm{W}$ boson and the LSP, the results probe top squarks with masses up to about $650 \mathrm{GeV}$ for LSP masses up to about $250 \mathrm{GeV}$.
\end{abstract}

\section{Introduction}

A search for the pair production of top squarks is performed using the pp collision dataset at a center-of-mass energy $\sqrt{s}=8 \mathrm{TeV}$ collected by the Compact Muon Solenoid (CMS) experiment [1] at the Large Hadron Collider (LHC) during 2012, corresponding to an integrated luminosity of $19.5 \mathrm{fb}^{-1}$. The gauge hierarchy problem has received additional attention following the recent discovery of a particle with mass of $\sim 125 \mathrm{GeV}$ consistent with the Higgs boson [2-4]. Relatively light top squarks, with masses below several hundred $\mathrm{GeV}$, are necessary if $\mathrm{Su}-$ persymmetry (SUSY) is to be the natural solution to the gauge hierarchy problem [5-9]. Searches for top squarks have also been performed by the ATLAS collaboration at the LHC [10-14], and by the CDF [15] and DØ [16] collaborations at the Tevatron. The analysis described here is an extension of a previous analysis [17] and more details can be found in reference [18].

The search presented here focuses on a R-parity conserving scenario, where SUSY particles are pair-produced, and the lightest SUSY particle (LSP) is stable and is a candidate dark matter particle. The lightest neutralino $\tilde{\chi}_{1}^{0}$ is considered to be the LSP, which escapes without detection. Two decay modes of the top squark ( $\mathfrak{t})$ are examined: $\tilde{\mathrm{t}} \rightarrow \mathrm{t} \tilde{\chi}_{1}^{0}$ and $\tilde{\mathrm{t}} \rightarrow \mathrm{b} \tilde{\chi}_{1}^{+} \rightarrow \mathrm{b} W^{+} \tilde{\chi}_{1}^{0}$, which are expected to have large branching fractions if kinematically accessible. Figure 1 shows the two signal processes of interest: pp $\rightarrow \widetilde{\mathrm{tt}}^{*} \rightarrow \bar{t} \bar{\chi} \tilde{\chi}_{1}^{0} \tilde{\chi}_{1}^{0} \rightarrow b \bar{b} W^{+} W^{-} \tilde{\chi}_{1}^{0} \tilde{\chi}_{1}^{0}$ and $\mathrm{pp} \rightarrow \tilde{\mathrm{tt}}^{*} \rightarrow b \bar{b} \tilde{\chi}_{1}^{+} \tilde{\chi}_{1}^{-} \rightarrow b \bar{b} W^{+} W^{-} \tilde{\chi}_{1}^{0} \tilde{\chi}_{1}^{0}$.

\footnotetext{
a e-mail: vimartin@fnal.gov

${ }^{b}$ Operated by Fermi Research Alliance, LLC under Contract No. DeAC02-07CH11359 with the United States Department of Energy.
}

\section{Event selection}

The analysis is based on events where one of the $W$ bosons decays leptonically and the other hadronically. The resulting signature includes one isolated lepton, four jets, two of which originate from $b$ quarks, and large missing transverse energy $\left(E_{\mathrm{T}}^{\mathrm{miss}}\right)$ from the two neutralinos. Selected events are thus required to contain one high transverse momentum $\left(p_{\mathrm{T}}\right)$ isolated lepton (electron $e$ [19] or muon $\mu$ [20]) and no additional isolated tracks or hadronic tau candidates, which are indications of an additional lepton. The veto on additional leptons reduces the background from $t \bar{t}$ events in which both $W$ bosons decay leptonically ( $\mathrm{t} \overline{\mathrm{t}} \rightarrow \ell^{+} \ell^{-}$). Events are also required to contain at least four high- $p_{\mathrm{T}}$ jets, where at least one jet is consistent with containing the decay of a heavy-flavor hadron (" $b$ tagged" [21]). In addition, the $E_{\mathrm{T}}^{\mathrm{miss}}$, defined as the magnitude of the vector sum of the transverse momenta of all the energy in the event, is required to be $E_{\mathrm{T}}^{\text {miss }}>100 \mathrm{GeV}$.

Background events containing a single leptonically decaying $W$ boson are suppressed by requiring that the transverse mass, constructed from the lepton $p_{\mathrm{T}}$ and the $E_{\mathrm{T}}^{\text {miss }}$, is much larger than the $W$ mass, $M_{\mathrm{T}}>120 \mathrm{GeV}$. Additional kinematic variables are used to discriminate signal from background. A useful discriminant is the $M_{\mathrm{T} 2}^{W}$ variable [22], which is the minimum "mother" particle mass compatible with all the transverse momenta and massshell constraints for a $\overline{\mathrm{t}} \rightarrow \ell^{+} \ell^{-}$event where the second lepton is missed. This variable has an endpoint at the top mass for $\bar{t} \bar{t}$ events, while the signal does not satisfy such a constraint. Two topological variables distinguish signal topologies where the LSPs are recoiling against the visible objects in the event from background $t \bar{t}$ topologies where the two tops have high $p_{\mathrm{T}}$ and are recoiling against each other. The first is the minimum difference in azimuthal angle between the $E_{\mathrm{T}}^{\text {miss }}$ vector and the two highest $p_{\mathrm{T}}$ jets, 

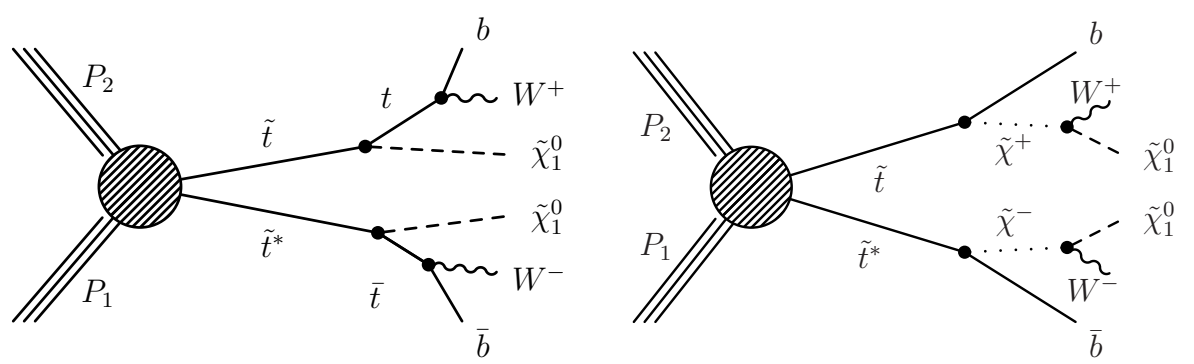

Figure 1. Diagram for top squark pair production for the $\tilde{\mathrm{t}} \rightarrow \mathrm{t} \tilde{\chi}_{1}^{0}$ (left) and $\tilde{\mathrm{t}} \rightarrow \mathrm{b} \tilde{\chi}_{1}^{+}$(right) decay modes.

which tends to be lower for $\mathrm{t} \overline{\mathrm{t}} \rightarrow \ell+$ jets events. The second variable is the $H_{\mathrm{T}}^{\text {ratio }}$, defined as the fraction of the scalar sum of the jet transverse energies $\left(H_{\mathrm{T}}\right)$ in the same hemisphere as the $E_{\mathrm{T}}^{\text {miss }}$, i.e., within $\pm 90^{\circ}$ of the $E_{\mathrm{T}}^{\text {miss }}$ vector, which is lower for signal events.

The dilepton $\bar{t} \bar{t}$ background is suppressed in the $\tilde{\mathrm{t}} \rightarrow$ $\mathrm{t} \tilde{\chi}_{1}^{0}$ search by searching for a combination of three of the jets in the event are consistent with a hadronic top decay $\mathrm{t} \rightarrow \mathrm{b} W \rightarrow \mathrm{bq} \overline{\mathrm{q}}$. The $p_{\mathrm{T}}$ spectrum of the leading b-tagged jet is also a useful discriminant in two signal scenarios. For the $\tilde{\mathrm{t}} \rightarrow \mathrm{b} \tilde{\chi}_{1}^{+}$mode, where the bottom quarks arise from the decay of the top squark, the bottom quark can be harder than the for $t \bar{t}$ events where the bottom quark comes from the decay of the top quark. For the $\tilde{\mathrm{t}} \rightarrow \mathrm{t} \tilde{\chi}_{1}^{0}$ decay mode where the top quark is off-shell, the $p_{\mathrm{T}}$ spectrum is softer than for the background where the top quark is on-shell. Finally an additional, related, discriminating variable is the geometrical separation between the leading b-tagged jet and the lepton.

Multiple signal regions (SRs) are defined in order to target the $\tilde{\mathrm{t}} \rightarrow \mathrm{t} \tilde{\chi}_{1}^{0}$ and $\tilde{\mathrm{t}} \rightarrow \mathrm{b} \tilde{\chi}_{1}^{+}$decay scenarios and different signal kinematics that depend on the region of parameter space defined by the SUSY particle masses. Two approaches are pursued to define SRs: an approach based on boosted decision tree (BDT) multivariate techniques and a cut-based approach. The cut-based SRs are defined by adding requirements on individual kinematic variables and a range of $E_{\mathrm{T}}^{\mathrm{miss}}$ requirements. The BDT combines the kinematic variables into a single discriminant, and the BDT SRs are defined by requirements on the value of this discriminant. Despite adding some complexity, the BDT approach improves the sensitivity of the search by up to $40 \%$. As a result, the primary result of the search is the BDT analysis, while the cut-based analysis serves as a cross-check.

In the BDT analysis, six SRs target the $\tilde{\mathrm{t}} \rightarrow \mathrm{t} \tilde{\chi}_{1}^{0}$ signal model and four SRs target the $\tilde{\mathrm{t}} \rightarrow \mathrm{b} \tilde{\chi}_{1}^{+}$signal model with a parameter $x=0.5$ that specifies the chargino mass, defined as $m_{\tilde{\chi}_{1}^{ \pm}}=x \cdot m_{\tilde{\mathfrak{t}}}+(1-x) \cdot m_{\tilde{\chi}_{1}^{0}}$. Additional $\tilde{\mathrm{t}} \rightarrow \mathrm{b} \tilde{\chi}_{1}^{+}$ signal regions targetting mass spectra with $x=0.25$ and 0.75 have also been pursued [18]. The search sensitivity decreases as the chargino and neutralino masses are closer to each other, or $x$ decreases.

\section{Backgrounds}

The SM backgrounds may be grouped into four categories, which in order of importance are: (1) $\mathrm{t} \overline{\mathrm{t}} \rightarrow \ell^{+} \ell^{-}$where one of the leptons is not identified, (2) $\bar{t} \bar{t}$ production in which one $\mathrm{W}$ boson decays leptonically and the other hadronically ( $\mathrm{t} \overline{\mathrm{t}} \rightarrow \ell+$ jets), as well as single-top production in the s- and t-channels, referred to as "single-lepton top", (3) a variety of SM processes with small cross sections, referred to as the "rare" processes, including $t \bar{t}$ events produced in association with a vector boson ( $(\overline{\mathrm{t}} \mathrm{W}, \overline{\mathrm{t}} \mathrm{Z}, \overline{\mathrm{t}} \gamma)$, processes with two $(W W, W Z, Z Z)$ and three $(W W W, W W Z, W Z Z$, $Z Z Z$ ) electroweak vector bosons, and single-top production in the tW-channel mode, and (4) production of $W$ bosons with jets $(W+$ jets). The multijet contribution to the background is negligible in the signal regions due to the requirement of a high- $p_{\mathrm{T}}$ isolated lepton, large $M_{\mathrm{T}}$, large $E_{\mathrm{T}}^{\text {miss }}$ and the presence of a b-tagged jet.

Backgrounds are estimated from MC simulations, normalized to the number of events in data in the transverse mass peak region, which is dominated by SM backgrounds. This minimizes systematic uncertainties associated with the knowledge of the $t \bar{t}$ production cross section, the integrated luminosity, the lepton efficiency, and jet energy scale. The simulation is validated in control regions (CRs) intended to enrich the data sample in specific background sources while maintaining kinematical properties that are similar to those in the SRs. Three CRs are used: a sample dominated by $\overline{\mathrm{t}} \rightarrow \ell^{+} \ell^{-}$events obtained by requiring the presence of two leptons (CR-2 $\ell$ ), a sample dominated by a mixture of $t \bar{t} \rightarrow \ell+$ jets and $t \bar{t} \rightarrow \ell^{+} \ell^{-}$ events obtained by requiring the presence of a lepton and an isolated track or hadronic tau candidate (CR- $\ell \mathrm{t})$ and a sample dominated by $W+$ jets events obtained by requiring no b-tagged jets in the event (CR-Ob).

The CRs are used to validate the modeling of the main kinematical variables in the analysis. In particular, the $M_{\mathrm{T}}$ distribution of the control sample after applying the SR-like requirements allows for a direct comparison of data and simulation. This provides a test of the ability of the method to correctly predict the SM background in the SRs. Figure 2 shows the $M_{\mathrm{T}}$ distribution in two control regions, $\mathrm{CR}-\ell \mathrm{t}$ and $\mathrm{CR}-0 \mathrm{~b}$, for events satisfying the BDT signal region requirement for the loosest (tightest) BDT SRs for the $\tilde{\mathrm{t}} \rightarrow \mathrm{t} \tilde{\chi}_{1}^{0}$ decay mode, targeting low (high) $\mathrm{m}_{\tilde{\mathrm{t}}}$ and small (large) mass differences $\Delta \mathrm{m}=\mathrm{m}_{\tilde{\mathrm{t}}}-\mathrm{m}_{\tilde{\chi}_{1}^{0}}$. Similar levels of agreement between data and MC are found 

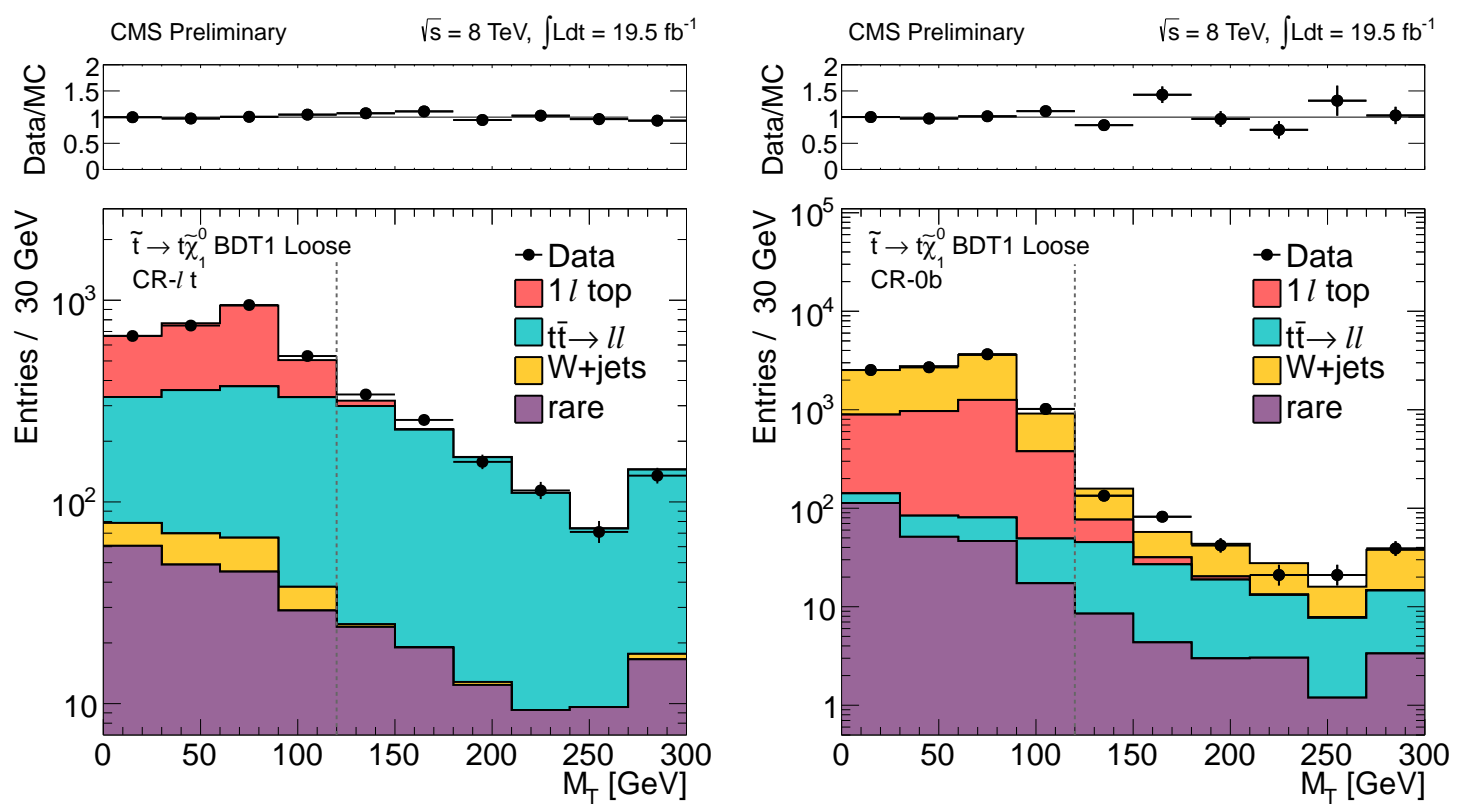

Figure 2. Data vs. MC simulation comparisons in the control regions CR- $\ell$ t (left), and CR-0b (right) of the $M_{\mathrm{T}}$ distribution after the signal-like requirement on the BDT discriminant is applied. The dashed lines in the right plots indicate the $M_{T}>120 \mathrm{GeV} \mathrm{SR}$ selection. For the plots in $\mathrm{CR}-0 \mathrm{~b}$, a scale factor is applied to the $\mathrm{MC}$ in the $M_{\mathrm{T}}$ tail. For all plots, the last bin contains the overflow.

for the other control regions. After normalizing to the $M_{\mathrm{T}}$ peak region, the number of events in data and simulation with $M_{\mathrm{T}}>120 \mathrm{GeV}$, indicated by the dashed line, can be directly compared. This comparison allows for the derivation of data/MC scale factors to be applied to the MC predictions. The only scale factor necessary is due to an underestimation of the $M_{\mathrm{T}}$ tail for single lepton top and $W+$ jets events.

In the case of $\mathrm{CR}-2 \ell$ and $\mathrm{CR}-\ell \mathrm{t}$, the number of data events with $M_{\mathrm{T}}>120 \mathrm{GeV}$ is consistent with the MC prediction. The level of agreement is used to assess a systematic uncertainty for the $\mathrm{t} \overline{\mathrm{t}} \rightarrow \ell^{+} \ell^{-}$background prediction. The uncertainty ranges from $5 \%$ for the loosest signal regions to $70 \%$ for the tightest signal regions, reflecting the limited statistical precision of the control samples after the SR-like requirements are applied. Finally, background contributions from rare processes are taken directly from the simulated samples, where their rates are normalized to the corresponding NLO cross sections.

\section{Results and Interpretation}

The background expectations and the corresponding data counts for BDT analysis for $\tilde{\mathrm{t}} \rightarrow \mathrm{t} \tilde{\chi}_{1}^{0}$ and $\tilde{\mathrm{t}} \rightarrow \mathrm{b} \tilde{\chi}_{1}^{+}$with $x=0.5$ are shown in Table 1 . Similar results are obtained for the $\tilde{\mathrm{t}} \rightarrow \mathrm{b} \tilde{\chi}_{1}^{+}$BDT analysis for the $x=0.25$ and 0.75 mass spectra and the cut-based analysis [18]. Figure 3 shows a comparison of data and simulation of the $M_{\mathrm{T}}$ distributions after all other requirements are applied for events satisfying the loosest and tightest $\tilde{\mathrm{t}} \rightarrow \mathrm{t} \tilde{\chi}_{1}^{0}$ BDT $\mathrm{SR}$ requirements. The SRs are to the right of the dotted line that indicates the cut value. The observed and predicted yields agree in all signal regions within about 1-1.5 standard deviations. Therefore, we observe no evidence for top-squark pair production. It may be noted that there is a tendency for the background predictions to lie somewhat above the observed yields; however the yields and background predictions in different signal regions are correlated.

The results of this search are interpreted in the context of the top squark pair production models where either $\tilde{\mathrm{t}} \rightarrow \mathrm{t} \tilde{\chi}_{1}^{0}$ or $\tilde{\mathrm{t}} \rightarrow \mathrm{b} \tilde{\chi}_{1}^{+} \rightarrow \mathrm{bW} \tilde{\chi}_{1}^{0}$, each with $100 \%$ branching fraction (see Fig. 1). The model is parametrized by the masses of the $\tilde{t}$ and the $\tilde{\chi}_{1}^{0}$. For each model point in the $m_{\tilde{t}}$ vs. $m_{\tilde{\chi}_{1}^{0}}$ parameter space, upper limits are set on the signal production cross section at $95 \%$ confidence level using the SR with the best expected sensitivity at that point. Exclusion regions are defined by comparing these cross section upper limits to the theoretical predictions for the signal cross sections. The results from the BDT analysis are shown in Fig. 4 (left) for the $\tilde{\mathrm{t}} \rightarrow \mathrm{t} \tilde{\chi}_{1}^{0}$ and $\tilde{\mathrm{t}} \rightarrow \mathrm{b} \tilde{\chi}_{1}^{+}$with $x=0.5$ scenarios. Depending on the model details, these results probe top squarks with masses between approximately 150 and $650 \mathrm{GeV}$ for $\tilde{\chi}_{1}^{0}$ with masses up to approximately $250 \mathrm{GeV}$. The results are not sensitive to the model points with $m_{\mathfrak{t}}-m_{\chi_{1}}=m_{\mathrm{t}}$ in the $\tilde{\mathrm{t}} \rightarrow \mathrm{t} \tilde{\chi}_{1}^{0}$ interpretation. In this case the $\tilde{\chi}_{1}^{0}$ is produced at rest in the top rest frame, resulting in low $E_{\mathrm{T}}^{\text {miss }}$ and low $M_{\mathrm{T}}$. However, this analysis is sensitive to signal scenarios with $m_{\mathfrak{t}}-m_{\chi_{1}}<m_{\mathrm{t}}$ and with top squarks lighter than the top, where the top quark in the decay $\tilde{\mathrm{t}} \rightarrow \mathrm{t} \tilde{\chi}_{1}^{0}$ is off-shell.

The polarizations of the final and intermediate state particles (top quarks in the $\tilde{\mathrm{t}} \rightarrow \mathrm{t} \tilde{\chi}_{1}^{0}$ scenario, and charginos and $W$ bosons in the $\tilde{t} \rightarrow b \tilde{\chi}_{1}^{+}$case) are model dependent and non-trivial functions of the top squark, chargino and neutralino mixing matrices $[23,24]$. The signal models 
Table 1. The result of the BDT analysis for $\tilde{\mathrm{t}} \rightarrow \mathrm{t} \tilde{\chi}_{1}^{0}$ and $\tilde{\mathrm{t}} \rightarrow \mathrm{b} \tilde{\chi}_{1}^{+} \rightarrow \mathrm{b} W^{+} \tilde{\chi}_{1}^{0}$ (with $m_{\tilde{\chi}_{1}^{ \pm}}=0.5 \cdot m_{\tilde{t}}+0.5 \cdot m_{\tilde{\chi}_{1}^{0}}$ ). For each signal region the individual background contributions, total background, and observed yields are indicated. The uncertainty includes both the statistical and systematic components.

\begin{tabular}{l||c|c|c|c|c|c||c|c|c|c}
\hline \multirow{2}{*}{ Sample } & \multicolumn{9}{c|}{$\tilde{\mathrm{t}} \rightarrow \mathrm{t} \tilde{\chi}_{1}^{0}$} & \multicolumn{4}{c}{$\tilde{\mathrm{t}} \rightarrow \mathrm{b} \tilde{\chi}_{1}^{+} \mathrm{x}=0.5$} \\
\cline { 2 - 11 } & BDT1 Loose & BDT1 Tight & BDT2 & BDT3 & BDT4 & BDT5 & BDT1 & BDT2 Loose & BDT2 Tight & BDT3 \\
\hline \hline $\mathrm{t} \tilde{\mathrm{t}} \rightarrow \ell \ell$ & $438 \pm 37$ & $68 \pm 11$ & $46 \pm 10$ & $5 \pm 2$ & $0.3 \pm 0.3$ & $48 \pm 13$ & $40 \pm 5$ & $21 \pm 4$ & $4 \pm 2$ & $6 \pm 2$ \\
$1 \ell$ Top & $251 \pm 93$ & $37 \pm 17$ & $22 \pm 12$ & $4 \pm 3$ & $0.8 \pm 0.9$ & $30 \pm 12$ & $24 \pm 10$ & $15 \pm 7$ & $4 \pm 3$ & $4 \pm 2$ \\
$W+$ jets & $27 \pm 7$ & $7 \pm 2$ & $6 \pm 2$ & $2 \pm 1$ & $0.8 \pm 0.3$ & $5 \pm 2$ & $5 \pm 1$ & $5 \pm 1$ & $2 \pm 1$ & $3 \pm 1$ \\
Rare & $47 \pm 23$ & $11 \pm 6$ & $10 \pm 5$ & $3 \pm 1$ & $1.0 \pm 0.5$ & $4 \pm 2$ & $8 \pm 4$ & $8 \pm 4$ & $3 \pm 1$ & $4 \pm 2$ \\
\hline Total & $763 \pm 102$ & $124 \pm 21$ & $85 \pm 16$ & $13 \pm 4$ & $2.9 \pm 1.1$ & $87 \pm 18$ & $77 \pm 12$ & $50 \pm 9$ & $13 \pm 4$ & $17 \pm 4$ \\
\hline Data & 728 & 104 & 56 & 8 & 2 & 76 & 67 & 35 & 12 & 13 \\
\hline
\end{tabular}
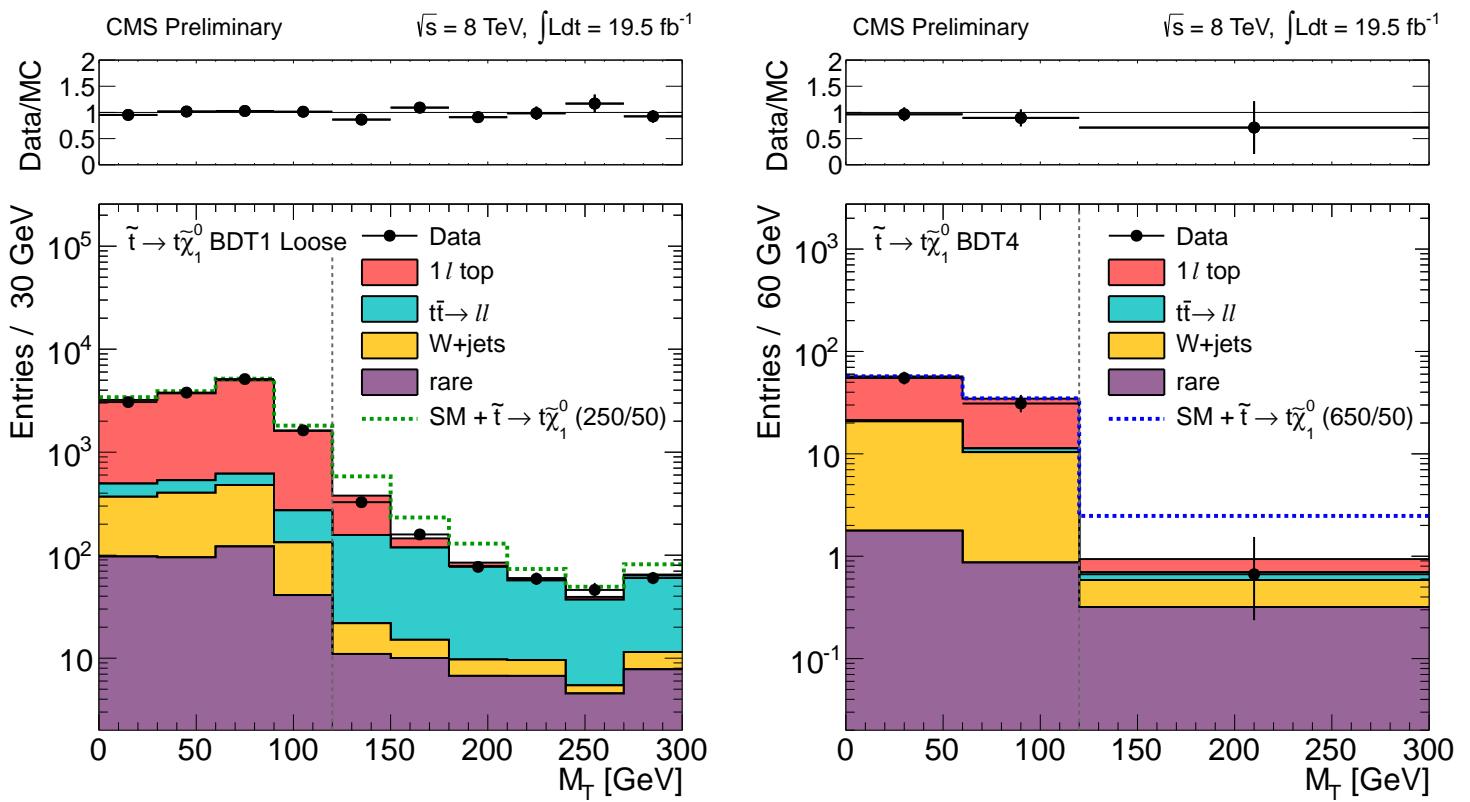

Figure 3. Comparison of the $M_{\mathrm{T}}$ distributions in data vs. MC simulation for events satisfying the loosest (left) and tightest (right) $\tilde{\mathrm{t}} \rightarrow \mathrm{t} \tilde{\chi}_{1}^{0}$ BDT signal region requirements. The distributions for the $\tilde{\mathrm{t}} \rightarrow \mathrm{t} \tilde{\chi}_{1}^{0}$ model with $m_{\tilde{t}}=250 \mathrm{GeV}$ and $m_{\tilde{\chi}_{1}^{0}}=50 \mathrm{GeV}$ (left) and $m_{\tilde{t}}=650 \mathrm{GeV}$ and $m_{\tilde{\chi}_{1}^{0}}=50 \mathrm{GeV}$ (right) are overlaid. The vertical dashed line indicates the SR requirement $\left(M_{\mathrm{T}}>120 \mathrm{GeV}\right)$. For the $M_{\mathrm{T}}$ distribution after the BDT4 requirement (right), the bin to the right of the vertical line contains all events with $M_{\mathrm{T}}>120 \mathrm{GeV}$, and has been scaled by $1 / 3$ to indicate the number of events per $60 \mathrm{GeV}$.

used in this interpretation are chosen to have no polarization. In order to examine the impact of this choice on the final result, the exclusion regions are compared under different assumptions. The exclusion regions obtained in the nominal $\tilde{\mathrm{t}} \rightarrow \mathrm{t} \tilde{\chi}_{1}^{0}$ scenario with unpolarized top quarks are compared to those obtained with pure left-handed and pure right-handed top quarks in Fig. 4 (top right). In the $\tilde{\mathrm{t}} \rightarrow \mathrm{b} \tilde{\chi}_{1}^{+}$scenario, the acceptance depends on the polarization of the chargino, and on the nature of the $W \tilde{\chi}_{1}^{0} \tilde{\chi}_{1}^{ \pm}$ coupling (left- or right-handed). In the nominal interpretation for the $\tilde{\mathrm{t}} \rightarrow \mathrm{b} \tilde{\chi}_{1}^{+}$model presented in Fig. 4, the signal events have an unpolarized chargino and a left/rightsymmetric $W \tilde{\chi}_{1}^{0} \tilde{\chi}_{1}^{ \pm}$coupling. A comparison of various limiting cases, shown in Fig. 4, indicates that the scenarios in which the results deviate the most from nominal correspond to right-handed charginos with either a right-handed $W \tilde{\chi}_{1}^{0} \tilde{\chi}_{1}^{ \pm}$coupling (maximum sensitivity) or a left-handed $W \tilde{\chi}_{1}^{0} \tilde{\chi}_{1}^{ \pm}$coupling (minimum sensitivity). These studies show that for both decay modes, the limits on the top squark and $\tilde{\chi}_{1}^{0}$ masses vary by $\pm 10-20 \mathrm{GeV}$ depending on the polarization assumption.

\section{Summary}

A search for the direct pair production of top squark pairs in the final state of a single isolated lepton, multiple jets, large $E_{\mathrm{T}}^{\text {miss }}$ and large $M_{\mathrm{T}}$ has been performed using the CMS pp collision dataset at $\sqrt{s}=8 \mathrm{TeV}$. Signal regions are defined with various kinematic discriminants targeting a range of signal models and kinematics. No significant excess is observed above the background predictions within the assessed uncertainties. The results are used to place constraints on the masses of top squarks in the mass range up to about $650 \mathrm{GeV}$, relevant for the natural SUSY solution to the gauge hierarchy problem.

\section{References}

[1] CMS Collaboration, J. Inst 3, S08004 (2008) . 

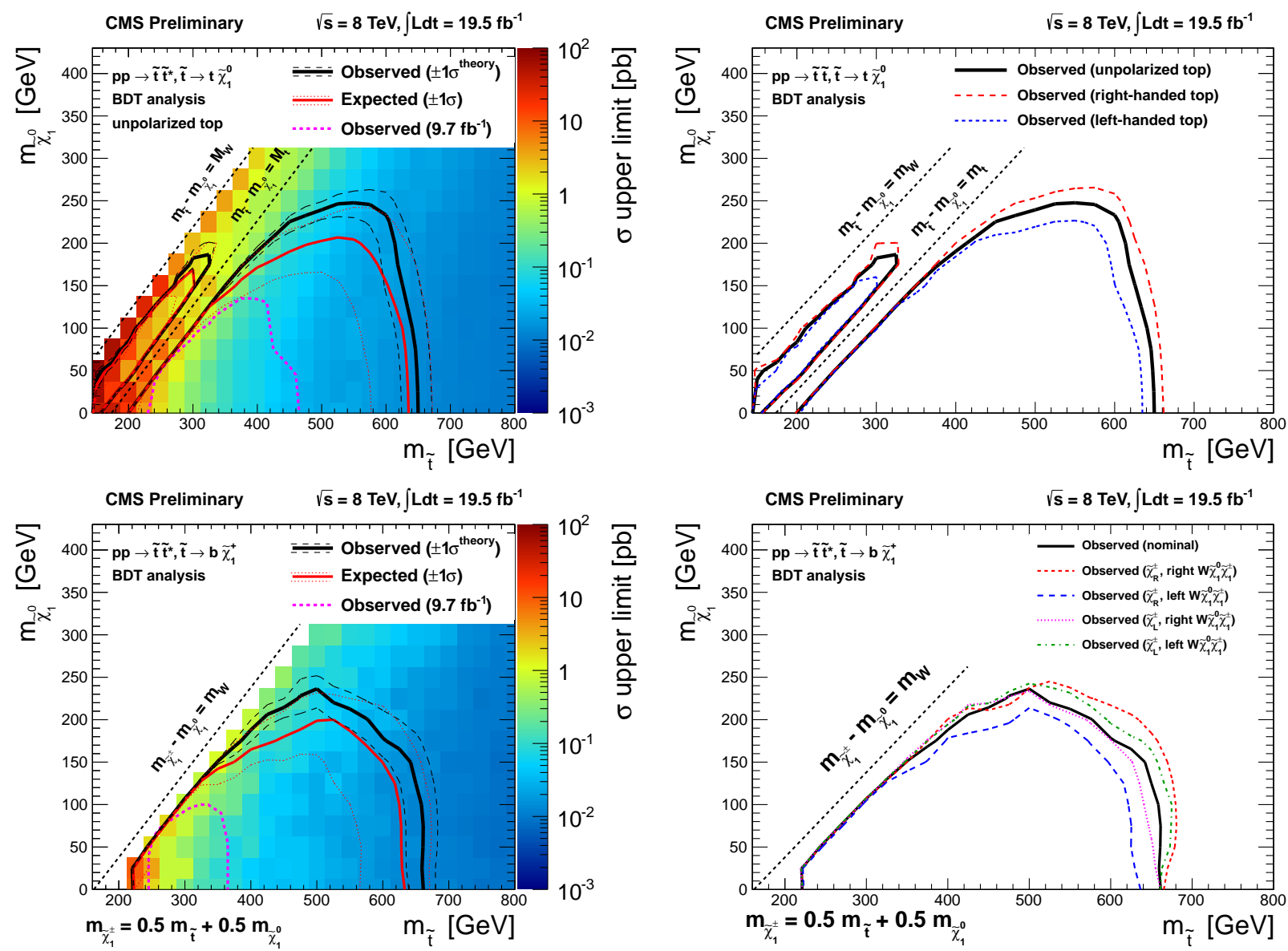

Figure 4. Interpretations using the primary results from the BDT method for the (top left) $\tilde{\mathrm{t}} \rightarrow \mathrm{t} \tilde{\chi}_{1}^{0}$ model, and for the $\tilde{\mathrm{t}} \rightarrow \mathrm{b} \tilde{\chi}_{1}^{+}$ model with chargino mass parameter $x=0.25$ (bottom left). The color scale indicates the observed cross section upper limit. The observed, median expected and \pm 1 standard deviation $(\sigma)$ expected exclusion contours are indicated. The right distributions compare the exclusion regions for different assumptions of the polarization of the intermediate decay particles, showing the observed excluded regions for the $\tilde{\mathrm{t}} \rightarrow \mathrm{t} \tilde{\chi}_{1}^{0}$ model for the case of unpolarized top quarks, right-handed top quarks, and left-handed top quarks (top right) and the observed excluded regions for the $\tilde{\mathrm{t}} \rightarrow \mathrm{b} \tilde{\chi}_{1}^{+}$model with $x=0.5$ for the nominal scenario, right-handed $\left(\tilde{\chi}_{R}^{ \pm}\right)$vs. left-handed $\left(\tilde{\chi}_{L}^{ \pm}\right)$ charginos, and right-handed vs. left-handed $W \tilde{\chi}_{1}^{0} \tilde{\chi}_{1}^{ \pm}$couplings (bottom right).

[2] M. W. Cahill-Rowley et al., Phys. Rev. D 86, 075015 (2012).

[3] CMS Collaboration, Phys. Lett. B 716, 30 (2012).

[4] ATLAS Collaboration, Phys. Lett. B 716, 1 (2012).

[5] R. Barbieri and G. Giudice, Nucl. Phys. B 30663 (1988).

[6] B. de Carlos and J. Casas, Phys. Lett. B 309 no. 3-4, 320 (1993).

[7] S. Dimopoulos and G. Giudice, Phys. Lett. B 357 no. 4, 573 (1995).

[8] R. Barbieri, G. Dvali, and L. J. Hall, Phys. Lett. B 377, 76 (1996).

[9] M. Papucci, J. T. Ruderman, and A. Weiler, JHEP 09, 035 (2012).

[10] ATLAS Collaboration, Phys. Rev. Lett. 109211802 (2012).

[11] ATLAS Collaboration, Phys. Rev. Lett. 109, 211803 (2012).
[12] ATLAS Collaboration, Eur. Phys. J. C72, 2237 (2012).

[13] ATLAS Collaboration, Phys. Lett. B720, 13 (2013).

[14] ATLAS Collaboration, JHEP 11, 094 (2012).

[15] CDF Collaboration, Phys. Rev. D 82092001 (2010).

[16] D0 Collaboration, Phys. Lett. B 696, 321 (2011).

[17] CMS Collaboration, CMS Physics Analysis Summary CMS-PAS-SUS-12-023, (2012).

[18] CMS Collaboration, CMS Physics Analysis Summary CMS-PAS-SUS-13-011, (2013).

[19] CMS Collaboration, CMS Physics Analysis Summary CMS-PAS-EGM-10-004, (2010).

[20] CMS Collaboration, CMS Physics Analysis Summary CMS-PAS-MUO-10-002, (2010).

[21] CMS Collaboration, JINST 8, P04013 (2013).

[22] Y. Bai et al., JHEP 07, 110 (2012).

[23] M. Perelstein and A. Weiler, JHEP 03141 (2009).

[24] I. Low, arXiv: 1304.0491. 